\title{
Population density, multiple harvesting, and ability of Ipomoea reptans to compete with native weeds at tropical wetlands
}

\author{
BENYAMIN LAKITAN ${ }^{1,2, \boldsymbol{\varphi}}$, KARTIKA KARTIKA ${ }^{1}$ \\ ${ }^{1}$ Faculty of Agriculture, Universitas Sriwijaya. Jl. Raya Palembang-Prabumulih Km 32, Indralaya, Ogan Ilir 30662, South Sumatra, Indonesia. \\ Tel.: +62-711-580169, Fax.: +62-711-580276, `email: blakitan60@unsri.ac.id \\ ${ }^{2}$ Research Center for Sub-optimal Lands, Universitas Sriwijaya. Jl. Padang Selasa No. 524, Palembang 30139, South Sumatra, Indonesia
}

Manuscript received: 18 August 2020. Revision accepted: 28 August 2020.

\begin{abstract}
Lakitan B, Kartika K. 2020. Population density, multiple harvesting, and ability of Ipomoea reptans to compete with native weeds at tropical wetlands. Biodiversitas 21: 4376-4383. Despite as a nutritious, fast-growing, and well-adapted leafy vegetable at tropical wetlands; Ipomoea reptans has not been intensively cultivated yet. This study was designed for increasing productivity of this vegetable by optimizing population density, extending harvesting period, and its ability to compete with native weeds at tropical wetlands. Bottom wet culture system (BWCS) was implemented by placing all pots within $2 \mathrm{~m}$ x 4 m experimental pool filled with water to 2-cm depth to make sure bottom part of the substrate within each pot was continuously water-saturated. Results of this study indicated that despite fluctuated yield at each harvest, accumulative yields after five consecutive harvests were not significantly different among population densities from 14 to 71 plants per $\mathrm{m}^{2}$. Yet, quality of yield in most cases was better in lower population density treatment (14 plants per $\mathrm{m}^{2}$ ), as indicated by SPAD value and marketable size of individual plants. Frequent NPK fertilizer application was effective for increasing yield. The first harvest was done at 4 weeks after seed sowing; thereafter, the plants were routinely reharvested at about every week. This fast-growing vegetable also exhibited ability to compete with native weeds commonly found at tropical wetlands at density up to $11.3 \mathrm{mg} \mathrm{cm}^{-2}$.
\end{abstract}

Keywords: bottom wet culture, Ipomoea reptans, plant-weed competition, population density

Abbreviations: BWCS: bottom wet culture system; DAS: days after sowing; NPK: nitrogen-phosphorous-potassium; SPAD: index for leaf chlorophyll content; WAS: weeks after sowing

\section{INTRODUCTION}

Ipomoea reptans is commonly named as water spinach in English and has been cultivated as leafy vegetable in many Asian countries (Hong and Gruda 2020); however, it has not been intensively cultivated. In Southeast Asia, this plant is popularly known as kangkong. Water spinach is an amphibious plant, adapting well to wetland ecosystems. It can float on water surface, grown on muddy soils, or on non-flooded lowlands. Besides as green vegetables, water spinach has been used in pharmaceutical industry (Chen et al. 2018; Hefny-Gad et al. 2018) and for phytoremediation of contaminated soil, especially in wetland ecosystem (Chanu and Gupta 2016; Tang et al. 2017). Water spinach is rich in amino acids and vitamins. It gives benefits via antioxidant and $\alpha$-glucosidase inhibitory activities (Lawal et al. 2017).

Water spinach is a fast-growing leafy vegetable. This vegetable can be cultivated in many ways. Farmers in Indonesia mostly grow water spinach by directly broadcast the seeds on lengthy rectangular raised beds. The plants are harvested at once every month and then immediately replanted again. Water spinach can also be planted in wider spaced rows, in this case, the first harvest is conducted within a month after planting and thereafter re-harvested regularly every week if water and nutrients availability are well managed. The other way is by free-floating stem cuttings on water. Water spinach has hollow stems, make it float on water (Kaur et al. 2016). Water spinach has also been used for purifying water polluted with some agrochemicals (Zhang et al. 2014); before the water was used for fish cultures, such as tilapia (Guo et al. 2019) and comet goldfish (Saaid et al. 2013) in an aquaponic system.

Multiple harvest system relies on rapid plant regrowth after each of previous harvest. Many crops have been cultivated with multiple harvest system. Fresh stalk biomass of sweet sorghum could be harvested three times annually, i.e. the first cut and two ratoon crops (Rolz et al. 2017). The stalk is used as raw material for ethanol production. Rice crops could be harvested twice, i.e. main harvest and ratoon crop (Wang et al. 2020). Sugarcane was commonly harvested several times over a stretch of several consecutive years (Marin et al. 2019). Multiple harvests in Gramineae genus or Cerealia crops have been more commonly practiced.

Meanwhile, multiple harvests in annual vegetables are rarely exercised. Fertilizer application after harvest is expected to enhance growth of the axillary stems in water spinach. Competition between water spinach at different plant population densities and native weeds at tropical wetlands has not been intensively studied.

Objectives of this study were to increase productivity of water spinach by optimizing plant population density from 1 to 5 plants per pot, equivalent to $14,28,42,57$, and 71 
plants per $\mathrm{m}^{2}$, respectively; extending harvest at least up to the fifth consecutive harvest; and for evaluating ability of water spinach to compete with native weeds in limited growing space. Yield was not only evaluated based on quantity but also on quality of harvested leaves.

\section{MATERIALS AND METHODS}

\section{Plant materials and experimental procedure}

Water spinach used in this study was Light Green cultivar. Seeds were sown in seedling trays filled with soilmanure mix. At 10 days after sowing (DAS), seedlings were selected for uniformity. The relatively uniform seedlings were transplanted into $30-\mathrm{cm}$ diameter pots, equivalent to $707 \mathrm{~cm}^{2}$ surface area. Height of the pot is 30 $\mathrm{cm}$, but height of the soil-manure mix within each pot was set at $25 \mathrm{~cm}$. In this study, the BWCS was employed. The BWCS was developed after tolerable depth of water table had been recognized (Meihana et al. 2017; Lakitan et al. 2018; Susilawati and Lakitan 2019; Kriswantoro et al. 2020a,b). Immediately after transplanting, all pots were placed in $2 \mathrm{~m} \times 4 \mathrm{~m}$ outdoor experimental pool filled with water to depth of $2 \mathrm{~cm}$, for assuring water penetration into growing substrate via bottom holes of each pot.

Base fertilizer was applied at rate of $5 \mathrm{~g}$ per pot to each experimental unit. Plant protection from insect pests was done by following organic vegetable protocols. No disease symptom was observed throughout life cycle of the plant, form seed sowing to the last harvest. Substrate moisture at depth of $15 \mathrm{~cm}$ was daily measured using soil moisture meter (Lutron PMS-714; Lutron Electronics Inc., Coopersburg, PA, USA). This research simulated the raised-bed and multiple harvest cultivation systems.

The first harvest at 30 DAS was done by cutting the main stem at position two nodes above base of the plant, for allowing axillary stems to grow at the nodes. At the second harvest, each axillary stem was cut at two nodes above base position of the respected primary axillary stem. Thereafter, the next harvests followed a similar procedure. In water spinach, each node can arise more than one axillary stem. The axillary stems were consecutively harvested 5 times during period from 30 to $60 \mathrm{DAS}$, i.e. at $30,40,48,55$, and 60 , respectively. These dates of harvest were decided based on availability of the stems that had reached the standard marketable size, i.e. length of stem is at least $35 \mathrm{~cm}$. Additional NPK fertilizer at rate of $5 \mathrm{~g}$ per pot was applied during extended harvesting period, i.e. the sixth and seventh harvest for additional exclusive evaluation on plant response to NPK fertilizer. Frequent and continuous harvests prevented the plants from developing their reproductive organs.

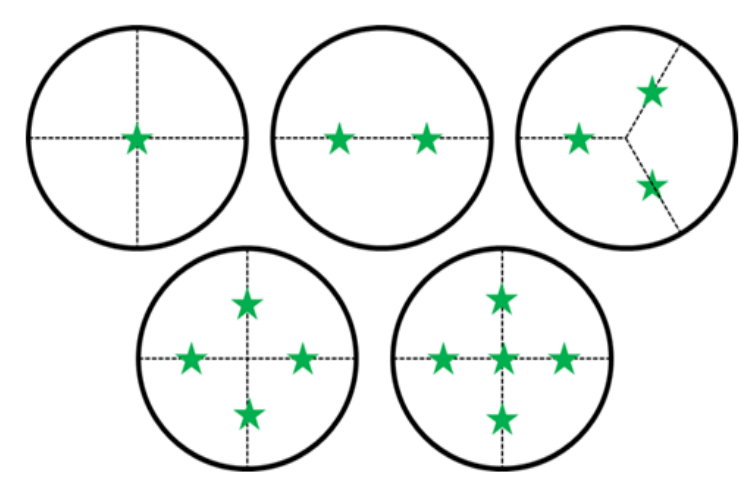

Figure 1. Arrangement position of Ipomoea reptans plants in each population density treatment. Star represents position of each plant.

Experimental units were arranged by following the Completely Random Design with 5 different population densities consisted of 5 replications for each treatment. Growth parameters were compared among population density treatments based on plant average within each treatment (for evaluating growth of individual plant) and compared based on total yield per pot, regardless of number of plants per pot (for evaluating productivity). Population densities evaluated were 1, 2, 3, 4, and 5 plants per pot, equivalent to $14,28,42,57$, and 71 plants per $\mathrm{m}^{2}$, respectively. Positions of each plant within pots for each population density treatments were arranged by placing them in specific pattern for creating more equally spaced position within each pot (Figure 1).

\section{Data collection}

Yield organs of water spinach are terminal and axillary stems which consist of young leaves, stem, and terminal bud. In this study, yield organ was divided into two components, i.e. (1) leaf blade and (2) stem, including terminal bud and petioles. Growth-associated data were collected at the same time with harvest data. Total stem and leaf fresh weight per pot; average stem and leaf fresh weight per plant; total leaf area per pot; average leaf area per plant; leaf SPAD value; leaf water content; dry weights of stem, leaf, and shoot per pot; and number of axillary stems per plant were collected during the first five harvests. Data for comparing growth and yield between plant grown on weed-free and weed-invested conditions were collected during the last four harvests, i.e. the fourth to seventh harvests based on total dry weight per pot data. SPAD value was measured using chlorophyll meter (KonicaMinolta SPAD 502 Plus) and leaf area was measured using digital image analyzer developed by Easlon and Bloom (2014). 


\section{Data analysis}

Growth and yield data were analyzed using the Analysis of Variance, followed by mean comparison among levels of treatment using the Least Squared Difference at $\mathrm{p}<0.05$ (LSD.05). Competition between water spinach and weeds was evaluated based on regression and correlation procedures.

\section{RESULTS AND DISCUSSION}

\section{Effects of population density on growth and yield}

Total of leaf and stem fresh weights is the yield of water spinach. The yield per individual plant was consistently higher at the lowest population density; conversely, lower yield per plant was found at the highest population density. However, total yield per pot was not consistent during harvesting period from 30 to 60 DAS (Table 1). Harvesting was conducted five times during the 30-day period. At the second and third harvests, yield of a single plant per pot was higher than average of multiple plants per pot. This yield difference was associated with faster growth of the single plant which occupying the whole spacious $707 \mathrm{~cm}^{2}$ growing space, compared to many plants per pot that competing for the same space.

Total accumulative yields for the first five harvests were not significantly different amongst all population density treatments; however, as expected due to competition amongst water spinach plants at high population density, average yield per individual plants was significantly low if population density was high and vice versa (Figure 2).

Leaf water content was not affected by plant population density in every harvest (Table 2). Despite differences in leaf area, leaf water content in all plants was relatively similar, or insignificantly different. Differences in water transpired by each plant were balanced with water uptake by their roots due to continuous availability of water within the rhizosphere. In bottom-wet culture, water can move upward into and within the rhizosphere due to capillarity or water potential difference. This culture system successfully provided water as required by hydrophilic water spinach plants, as proven by high leaf water content in each plant. There was no significant difference in leaf water content; therefore, stem, leaf, and shoot dry weight were following pattern of fresh weight.

Earlier or later, each main and axillary stem eventually reached the $35-\mathrm{cm}$ market standard before being harvested; therefore, higher number of harvested axillary stems also indicated faster growth of the specified plants. Single plant per pot produced axillary stems earlier and faster than multiple plants were grown per pot (Table 3). Axillary stems are important in water spinach, since axillary stems are the harvestable part of water spinach after they reach the marketable size.

\section{Response of water spinach to NPK fertilizer}

After the third harvest, NPK fertilizer at rate of $5 \mathrm{~g}$ per pot was applied to all experimental units as a non-treatment practice. Amazingly, at the fourth harvest, the most densely populated treatment, i.e. 5 plants per pot, regained status as the most productive treatment, i.e. the highest total yield per pot. This is a clear indication that soil nutrients were rapidly depleted if five plants grown in a single pot. In the fifth harvest, the highest yield was exhibited by treatment with four plants per pot. At this point, there is an indication that if five plants were grown in an area around $707 \mathrm{~cm}^{2}$, NPK fertilizer should be applied more frequently or at about every week, in order to achieve the highest possible yield.

Table 1. Fresh weight per pot and per plant at five harvests of Ipomoea reptans grown at 5 different population density

\begin{tabular}{|c|c|c|c|c|}
\hline \multirow{2}{*}{$\begin{array}{c}\text { Population } \\
\text { (plant per } \\
\text { pot) }\end{array}$} & \multicolumn{2}{|c|}{$\begin{array}{c}\text { Total fresh weight (g } \\
\text { per pot) }\end{array}$} & \multicolumn{2}{|c|}{$\begin{array}{c}\text { Fresh weight (g per } \\
\text { plant) }\end{array}$} \\
\hline & Stem & Leaf & Stem & Leaf \\
\hline & \multicolumn{4}{|c|}{ First harvest (30 DAS) } \\
\hline 1 & $5.58 \mathrm{~d}$ & $4.63 \mathrm{c}$ & $5.58 \mathrm{a}$ & $4.63 \mathrm{a}$ \\
\hline 2 & $8.31 \mathrm{~cd}$ & $6.36 \mathrm{c}$ & $4.16 \mathrm{~b}$ & $3.18 \mathrm{~b}$ \\
\hline 3 & $12.03 \mathrm{bc}$ & $9.49 \mathrm{~b}$ & $4.01 \mathrm{~b}$ & $3.16 \mathrm{~b}$ \\
\hline 4 & $14.59 \mathrm{ab}$ & $10.90 \mathrm{ab}$ & $3.65 \mathrm{~b}$ & $2.72 \mathrm{~b}$ \\
\hline 5 & $16.54 \mathrm{a}$ & $12.59 \mathrm{a}$ & $3.01 \mathrm{~b}$ & $2.51 \mathrm{~b}$ \\
\hline \multirow[t]{2}{*}{ LSD.05 } & 3.76 & 5.27 & 1.36 & 1.07 \\
\hline & \multicolumn{4}{|c|}{ Second harvest (40 DAS) } \\
\hline 1 & $13.26 \mathrm{a}$ & $11.55 \mathrm{a}$ & $13.26 \mathrm{a}$ & $11.55 \mathrm{a}$ \\
\hline 2 & $6.10 \mathrm{a}$ & $6.78 \mathrm{a}$ & $3.05 \mathrm{~b}$ & $3.39 \mathrm{~b}$ \\
\hline 3 & $5.70 \mathrm{a}$ & $5.88 \mathrm{a}$ & $1.90 \mathrm{~b}$ & $1.96 \mathrm{~b}$ \\
\hline 4 & $6.49 \mathrm{a}$ & $6.82 \mathrm{a}$ & $1.62 \mathrm{~b}$ & $1.70 \mathrm{~b}$ \\
\hline 5 & $7.46 \mathrm{a}$ & $7.90 \mathrm{a}$ & $1.49 \mathrm{~b}$ & $1.58 \mathrm{~b}$ \\
\hline \multirow{2}{*}{ LSD.05 } & \multirow{2}{*}{\multicolumn{4}{|c|}{$\begin{array}{c}6.88 \\
\text { Third harvest }(48 \text { DAS }) * 5.12\end{array}$}} \\
\hline & & & & \\
\hline 1 & $11.72 \mathrm{a}$ & $10.96 \mathrm{a}$ & $11.72 \mathrm{a}$ & $10.96 \mathrm{a}$ \\
\hline 2 & $9.33 \mathrm{ab}$ & $9.35 \mathrm{a}$ & $4.66 \mathrm{~b}$ & $4.68 \mathrm{~b}$ \\
\hline 3 & $9.81 \mathrm{ab}$ & $10.60 \mathrm{a}$ & 3.27 & $3.53 \mathrm{bc}$ \\
\hline 4 & $8.55 \mathrm{ab}$ & $9.90 \mathrm{a}$ & 2.14 & $2.48 \mathrm{~cd}$ \\
\hline 5 & $7.62 \mathrm{~b}$ & $8.69 \mathrm{a}$ & 1.52 & $1.74 \mathrm{~d}$ \\
\hline \multirow[t]{2}{*}{ LSD.05 } & 3.52 & & 2.07 & 1.77 \\
\hline & \multicolumn{4}{|c|}{ Forth harvest (55 DAS) } \\
\hline 1 & $8.00 \mathrm{c}$ & $8.09 \mathrm{~b}$ & $8.00 \mathrm{a}$ & $8.09 \mathrm{a}$ \\
\hline 2 & $10.66 \mathrm{bc}$ & $10.30 \mathrm{~b}$ & $5.33 \mathrm{ab}$ & $5.15 \mathrm{ab}$ \\
\hline 3 & $10.02 \mathrm{bc}$ & $10.20 \mathrm{~b}$ & $3.34 \mathrm{~b}$ & $3.40 \mathrm{~b}$ \\
\hline 4 & $15.49 \mathrm{ab}$ & $14.35 \mathrm{ab}$ & $3.87 \mathrm{~b}$ & $3.59 \mathrm{~b}$ \\
\hline 5 & $20.24 \mathrm{a}$ & $20.16 \mathrm{a}$ & $4.05 \mathrm{~b}$ & $4.03 \mathrm{~b}$ \\
\hline \multirow[t]{2}{*}{ LSD.05 } & 7.06 & 6.35 & & \\
\hline & \multicolumn{4}{|c|}{ Fifth harvest (60 DAS) } \\
\hline 1 & $8.18 \mathrm{a}$ & $9.46 \mathrm{a}$ & $8.18 \mathrm{a}$ & $9.46 \mathrm{a}$ \\
\hline 2 & $11.04 \mathrm{a}$ & $12.28 \mathrm{a}$ & $5.52 \mathrm{ab}$ & $6.14 \mathrm{ab}$ \\
\hline 3 & $10.34 \mathrm{a}$ & $11.87 \mathrm{a}$ & $3.45 \mathrm{bc}$ & $3.96 \mathrm{bc}$ \\
\hline 4 & $11.31 \mathrm{a}$ & $12.55 \mathrm{a}$ & $2.83 \mathrm{bc}$ & $3.14 \mathrm{bc}$ \\
\hline 5 & $8.31 \mathrm{a}$ & $10.37 \mathrm{a}$ & $1.66 \mathrm{c}$ & $2.07 \mathrm{c}$ \\
\hline LSD.05 & & & 2.90 & 3.40 \\
\hline
\end{tabular}




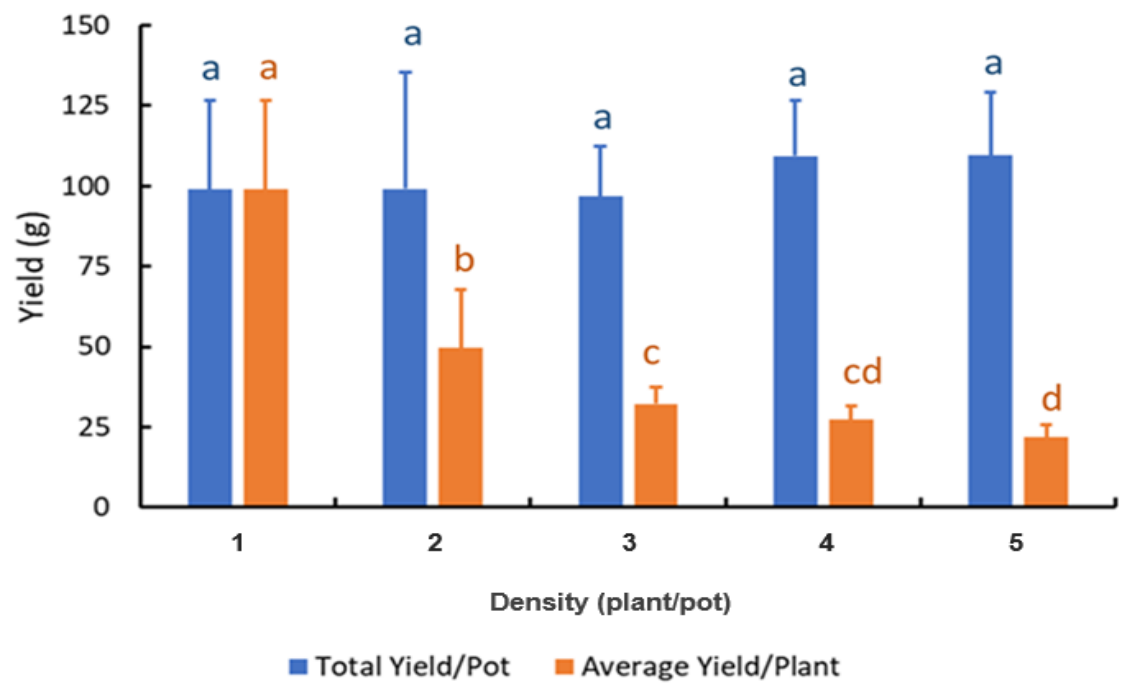

Figure 2. Accumulative yield per pot (blue bars) and average yield per plant (orange bars) in Ipomoea reptans after five harvests.

Table 2. Total leaf area per pot and per plant, leaf SPAD, and leaf water content at five harvests of Ipomoea reptans grown at 5 different population density

\begin{tabular}{|c|c|c|c|c|}
\hline \multirow{2}{*}{$\begin{array}{c}\text { Population } \\
\text { (plant per } \\
\text { pot) }\end{array}$} & \multicolumn{2}{|c|}{ Leaf area $\left(\mathrm{cm}^{2}\right)$} & \multirow[b]{2}{*}{$\begin{array}{c}\text { Leaf } \\
\text { SPAD }\end{array}$} & \multirow{2}{*}{$\begin{array}{c}\text { Leaf water } \\
\text { content } \\
(\%)\end{array}$} \\
\hline & $\begin{array}{l}\text { Total } \\
\text { per pot }\end{array}$ & $\begin{array}{l}\text { Average } \\
\text { per plant }\end{array}$ & & \\
\hline & \multicolumn{4}{|c|}{ First harvest (30 DAS) } \\
\hline 1 & $233.05 \mathrm{c}$ & $233.05 \mathrm{a}$ & $40.88 \mathrm{a}$ & $89.41 \mathrm{a}$ \\
\hline 2 & $323.08 \mathrm{c}$ & $161.54 \mathrm{~b}$ & $36.16 \mathrm{~b}$ & $89.38 \mathrm{a}$ \\
\hline 3 & $465.35 \mathrm{~b}$ & $155.12 \mathrm{~b}$ & $36.44 \mathrm{~b}$ & $89.16 \mathrm{a}$ \\
\hline 4 & $573.46 \mathrm{ab}$ & $143.37 \mathrm{~b}$ & $37.24 \mathrm{ab}$ & $88.73 \mathrm{a}$ \\
\hline 5 & $667.98 \mathrm{a}$ & $133.60 \mathrm{~b}$ & $35.10 \mathrm{~b}$ & $88.93 \mathrm{a}$ \\
\hline \multirow{2}{*}{ LSD.05 } & 118.01 & 45.63 & 4.16 & \\
\hline & \multicolumn{4}{|c|}{ Second harvest (40 DAS) } \\
\hline 1 & $583.70 \mathrm{a}$ & $583.70 \mathrm{a}$ & $36.42 \mathrm{a}$ & $88.07 \mathrm{a}$ \\
\hline 2 & $363.10 \mathrm{a}$ & $181.50 \mathrm{~b}$ & $35.62 \mathrm{ab}$ & $89.19 \mathrm{a}$ \\
\hline 3 & $322.20 \mathrm{a}$ & $107.40 \mathrm{~b}$ & $33.62 \mathrm{~b}$ & $87.65 \mathrm{a}$ \\
\hline 4 & $357.60 \mathrm{a}$ & $89.40 \mathrm{~b}$ & $35.00 \mathrm{ab}$ & $87.99 \mathrm{a}$ \\
\hline 5 & $420.00 \mathrm{a}$ & $84.00 \mathrm{~b}$ & $34.30 \mathrm{ab}$ & $88.45 \mathrm{a}$ \\
\hline \multirow[t]{2}{*}{ LSD.05 } & & 260.83 & 2.67 & \\
\hline & \multicolumn{4}{|c|}{ Third harvest (48 DAS) * } \\
\hline 1 & $553.67 \mathrm{a}$ & $553.67 \mathrm{a}$ & $37.50 \mathrm{a}$ & $85.01 \mathrm{a}$ \\
\hline 2 & $490.87 \mathrm{a}$ & $245.44 \mathrm{~b}$ & $37.46 \mathrm{a}$ & $85.58 \mathrm{a}$ \\
\hline 3 & $536.93 \mathrm{a}$ & $178.98 \mathrm{bc}$ & $37.44 \mathrm{a}$ & $84.89 \mathrm{a}$ \\
\hline 4 & $565.06 \mathrm{a}$ & $141.27 \mathrm{bc}$ & $34.90 \mathrm{~b}$ & $84.73 \mathrm{a}$ \\
\hline 5 & $430.85 \mathrm{a}$ & $86.17 \mathrm{c}$ & $35.18 \mathrm{~b}$ & $84.85 \mathrm{a}$ \\
\hline \multirow[t]{2}{*}{ LSD.05 } & & 105.47 & 2.15 & \\
\hline & \multicolumn{4}{|c|}{ Forth harvest (55 DAS) } \\
\hline 1 & $411.25 b$ & $411.25 \mathrm{a}$ & $42.36 \mathrm{a}$ & $89.65 \mathrm{a}$ \\
\hline 2 & $510.06 \mathrm{~b}$ & $255.03 \mathrm{ab}$ & $42.18 \mathrm{a}$ & 89.95 a \\
\hline 3 & $510.16 \mathrm{~b}$ & $170.05 \mathrm{~b}$ & $37.08 \mathrm{~b}$ & $91.60 \mathrm{a}$ \\
\hline 4 & $724.48 \mathrm{ab}$ & $181.12 \mathrm{~b}$ & $41.52 \mathrm{a}$ & $88.75 \mathrm{a}$ \\
\hline 5 & $1031.81 \mathrm{a}$ & $206.36 \mathrm{~b}$ & $40.54 \mathrm{ab}$ & $89.11 \mathrm{a}$ \\
\hline \multirow{2}{*}{ LSD.05 } & 361.77 & 201.18 & 3.62 & \\
\hline & \multicolumn{4}{|c|}{ Fifth harvest (60 DAS) } \\
\hline 1 & $503.12 \mathrm{a}$ & $503.12 \mathrm{a}$ & $38.94 \mathrm{a}$ & $88.31 \mathrm{a}$ \\
\hline 2 & $667.28 \mathrm{a}$ & $333.64 \mathrm{ab}$ & $39.30 \mathrm{a}$ & $88.44 \mathrm{a}$ \\
\hline 3 & $645.97 \mathrm{a}$ & $215.32 \mathrm{bc}$ & $38.88 \mathrm{a}$ & $88.42 \mathrm{a}$ \\
\hline 4 & $679.42 \mathrm{a}$ & $169.85 \mathrm{bc}$ & $38.36 \mathrm{a}$ & $88.36 \mathrm{a}$ \\
\hline 5 & $563.67 \mathrm{a}$ & $112.73 \mathrm{c}$ & $38.26 \mathrm{a}$ & $88.70 \mathrm{a}$ \\
\hline LSD. 05 & & 182.57 & & \\
\hline
\end{tabular}

*) NPK fertilizer application at rate of $5 \mathrm{~g}$ per pot
Table 3. Stem, leaf, and shoot dry weight and number of harvestable stems at five harvests of Ipomoea reptans grown at 5 different population density

\begin{tabular}{|c|c|c|c|c|}
\hline \multirow{2}{*}{$\begin{array}{c}\text { Population } \\
\text { (plant per } \\
\text { pot) }\end{array}$} & \multicolumn{3}{|c|}{ Dry weight (g per pot) } & \multirow{2}{*}{$\begin{array}{c}\text { Total } \\
\text { harvestable } \\
\text { stems per pot }\end{array}$} \\
\hline & Stem & Leaf & Shoot & \\
\hline & \multicolumn{4}{|c|}{ First harvest (30 DAS) } \\
\hline 1 & $0.30 \mathrm{~d}$ & $0.51 \mathrm{c}$ & $0.80 \mathrm{c}$ & $3.2 \mathrm{a}$ \\
\hline 2 & $0.47 \mathrm{~cd}$ & $0.68 \mathrm{c}$ & $1.14 \mathrm{c}$ & $1.3 \mathrm{~b}$ \\
\hline 3 & $0.70 \mathrm{bc}$ & $1.02 \mathrm{~b}$ & $1.72 \mathrm{~b}$ & $1.93 \mathrm{ab}$ \\
\hline 4 & $0.85 \mathrm{ab}$ & $1.23 \mathrm{ab}$ & $2.08 \mathrm{ab}$ & $1.95 \mathrm{ab}$ \\
\hline 5 & $1.02 \mathrm{a}$ & $1.40 \mathrm{a}$ & $2.42 \mathrm{a}$ & $1.04 \mathrm{~b}$ \\
\hline \multirow[t]{2}{*}{ LSD.05 } & 0.24 & 0.29 & 0.52 & 1.27 \\
\hline & \multicolumn{4}{|c|}{ Second harvest (40 DAS) } \\
\hline 1 & $0.97 \mathrm{a}$ & $1.5 \mathrm{a}$ & $2.47 \mathrm{a}$ & $2.80 \mathrm{a}$ \\
\hline 2 & $0.43 \mathrm{~b}$ & $0.77 \mathrm{~b}$ & $1.2 \mathrm{~b}$ & $2.20 \mathrm{a}$ \\
\hline 3 & $0.45 \mathrm{~b}$ & $0.73 \mathrm{~b}$ & $1.18 \mathrm{~b}$ & $2.80 \mathrm{a}$ \\
\hline 4 & $0.46 \mathrm{~b}$ & $0.81 \mathrm{~b}$ & $1.27 \mathrm{~b}$ & $3.40 \mathrm{a}$ \\
\hline 5 & $0.56 \mathrm{~b}$ & $0.95 \mathrm{~b}$ & $1.51 \mathrm{~b}$ & $3.00 \mathrm{a}$ \\
\hline \multirow[t]{2}{*}{ LSD.05 } & 0.39 & 0.48 & 0.73 & \\
\hline & \multicolumn{4}{|c|}{ Third harvest (48 DAS) * } \\
\hline 1 & $0.95 \mathrm{a}$ & $1.65 \mathrm{a}$ & $2.60 \mathrm{a}$ & $4.40 \mathrm{ab}$ \\
\hline 2 & $0.76 \mathrm{a}$ & $1.36 \mathrm{a}$ & $2.12 \mathrm{a}$ & $4.00 \mathrm{~b}$ \\
\hline 3 & $0.91 \mathrm{a}$ & $1.60 \mathrm{a}$ & $2.52 \mathrm{a}$ & $6.00 \mathrm{ab}$ \\
\hline 4 & $0.83 \mathrm{a}$ & $1.51 \mathrm{a}$ & $2.34 \mathrm{a}$ & $6.20 \mathrm{a}$ \\
\hline 5 & $0.77 \mathrm{a}$ & $1.32 \mathrm{a}$ & $2.09 \mathrm{a}$ & $5.20 \mathrm{ab}$ \\
\hline \multirow[t]{2}{*}{ LSD.05 } & \multirow{2}{*}{\multicolumn{4}{|c|}{ Forth harvest (55 DAS) }} \\
\hline & & & & \\
\hline 1 & $0.41 \mathrm{c}$ & $0.86 \mathrm{~b}$ & $1.27 \mathrm{~b}$ & $3.00 \mathrm{c}$ \\
\hline 2 & $0.55 \mathrm{bc}$ & $1.10 \mathrm{~b}$ & $1.65 \mathrm{~b}$ & $3.80 \mathrm{c}$ \\
\hline 3 & $0.52 \mathrm{bc}$ & $0.84 \mathrm{~b}$ & $1.36 \mathrm{~b}$ & $5.60 \mathrm{bc}$ \\
\hline 4 & $0.83 \mathrm{ab}$ & $1.61 \mathrm{ab}$ & $2.44 \mathrm{ab}$ & $7.00 \mathrm{~b}$ \\
\hline 5 & $1.10 \mathrm{a}$ & $2.22 \mathrm{a}$ & $3.32 \mathrm{a}$ & $10.40 \mathrm{a}$ \\
\hline \multirow[t]{2}{*}{ LSD.05 } & 0.39 & 0.83 & 1.19 & 2.69 \\
\hline & \multicolumn{4}{|c|}{ Fifth harvest (60 DAS) } \\
\hline 1 & $0.50 \mathrm{a}$ & $1.14 \mathrm{a}$ & $1.64 \mathrm{a}$ & $4.60 \mathrm{c}$ \\
\hline 2 & $0.63 \mathrm{a}$ & $1.41 \mathrm{a}$ & $2.03 \mathrm{a}$ & $6.80 \mathrm{ab}$ \\
\hline 3 & $0.63 \mathrm{a}$ & $1.36 \mathrm{a}$ & $1.98 \mathrm{a}$ & $6.60 \mathrm{bc}$ \\
\hline 4 & $0.69 \mathrm{a}$ & $1.45 \mathrm{a}$ & $2.14 \mathrm{a}$ & $8.80 \mathrm{a}$ \\
\hline 5 & $0.49 \mathrm{a}$ & $1.18 \mathrm{a}$ & $1.67 \mathrm{a}$ & $7.00 \mathrm{ab}$ \\
\hline LSD.05 & & & & 2.08 \\
\hline
\end{tabular}


The highest total leaf area per plant was consistently observed in single plant per pot at every harvest. Similar trend was also observed on SPAD value, except for the fifth harvest. Therefore, single plant was not only growing more vigorously but also greener. Meanwhile, at the highest plant density treatment, total leaf area was responded to NPK fertilizer application at a week before targeted harvesting, as indicated by significantly higher total leaf area at the fourth harvests (Table 2).

NPK fertilizer application effectively increased number of axillary stems as shown in the fourth harvest (Table 3 ). Increase in total fresh yield (Table 1) and dry weight per pot at a week after NPK fertilizer application was associated with increase in total number of harvestable axillary stems.

Effectiveness of NPK fertilizer application at rate of $5 \mathrm{~g}$ per pot at a week prior to harvest was only short-lasting in densely populated treatment, i.e. 5 plants per pot. After abrupt increase of total yield at the following week after fertilization, the total yield dropped back to level similar to single plant per pot and other population density treatments. The NPK effect was repeatable as shown at Figure 3. Application of NPK at similar rate after the fifth harvest also increased shoot dry weight of water spinach grown at high population density at the sixth harvest, but at the following week (the seventh harvest), the yield leveled back with the yield of all other plant density treatments, similar to case of fertilizer application after the third harvest (Figure 3).

\section{Dominance of water spinach over native weeds}

In some reported cases, weeds reduced yield of agricultural crops since they competed for water, nutrient, light, and growing space. However, weed density at rate up to $11.3 \mathrm{mg} \mathrm{cm}^{-2}$ (weed dry weight per surface area) did not directly affect yield of water spinach and there was no significant correlation between yield of water spinach grown under weedy and weed-free condition (Figure 4). Water spinach grew rigorously and fast. It took only 30 days to harvest after planting. In most cases, water spinach germinated earlier and grew faster compared to native weeds at tropical wetlands.

Total shoot dry weight per pot was heavier in higher population density treatment. The total dry weight at density of 4 or 5 plants per pot was significantly higher than those at density of 1,2 , or 3 plants per pot. Heavier total dry weight in higher population density was partly associated with suppression of densely populated water spinach on growth of native weed (Figure 5). However, total accumulative yields for five harvests were not significantly different across all population density treatment as mentioned in Figure 4. Total roots dry weight per pot exhibited similar pattern as total shoot dry weight. Higher total roots dry weight per pot was observed at higher density treatments of 4 and 5 plants per pot. Reversely, longest roots were found in single plant per pot treatment (Figure 6).

\section{Discussion}

Substrate surface area within pot used in this study was $707 \mathrm{~cm}^{2}$ and the plants were about equally spaced (Figure 1) therefore, densities of $1,2,3,4$, and 5 plants per pot were equivalent to $14,28,42,57$, and 71 plants per $\mathrm{m}^{2}$, respectively. Results of this study indicated that highest total yield was achieved at the highest population density of 71 plants per $\mathrm{m}^{2}$ at the first harvest and the other harvests immediately a week after NPK fertilizers were applied; however, lowest density at 14 plants per $\mathrm{m}^{2}$ consistently yielded highest fresh weight per individual plant (Table 1). Selamat et al. (2012) also reported that water spinach cultivated at lower densities $(\leq 55$ plants per $\mathrm{m}^{2}$ ) produced better quality yield than those cultivated at higher densities $\left(\geq 112\right.$ plants per $\left.\mathrm{m}^{2}\right)$. Similar trend was reported by Sarkar et al. (2014) regarding effects of planting density on yield in water spinach. Cultivation at lower density produced larger individual plants but lower total yield per unit area.

Dense population caused low-quality plants, i.e. slimmer stem due to etiolation and smaller leaves, caused by competition amongst plants for water, nutrients, and sunlight. Ahmed et al. (2013) found that at a similar population density, yield was higher in water spinach directly exposed to full sunlight than those grown under lower light intensity. Therefore, water spinach can be classified as sun plant.

There are several systems used for water spinach cultivation (Enriquez 2017). This experiment was designed for multiple harvests on weekly basis up to seven harvests, starting in the fourth week. After each harvest, the plants developed axillary stems that can be harvested at the following week if the stems had reached marketable size ( 25 to $30 \mathrm{~cm}$ long, measured from cutting position). Plants occupied larger areas (lower density) developed more axillary stems per plant (Table 3) and grew faster as indicated by increase of yield fresh weight per plant (Table 1 ); therefore, they could be harvested at 5 days after every previous harvest. Meanwhile, densely populated plants required full of 7 days to harvest.

It was unexpected that cumulative yields after five harvests were almost similar for all population density treatments (Figure 1). This fact led to conclusion that it was better to grow water spinach in multiple harvest cultivation system using one seedling per pot or with population density of 14 plants per $\mathrm{m}^{2}$. Low-density planting produced better quality, less seeds, and seedlings to start with, and can be more frequently harvested.

Axillary stems were developed more and earlier in single plants per pot than multiple plants were grown per pot. Axillary stems are important in water spinach, since axillary stems are the next harvestable part of water spinach after they reach marketable size. Some other crops behaved similarly to water spinach; for instance, Rondanini et al. (2017) reported that low plant density was compensated by strong vegetative plasticity, including producing more axillary stems in well-watered and fertilized rapeseed. 


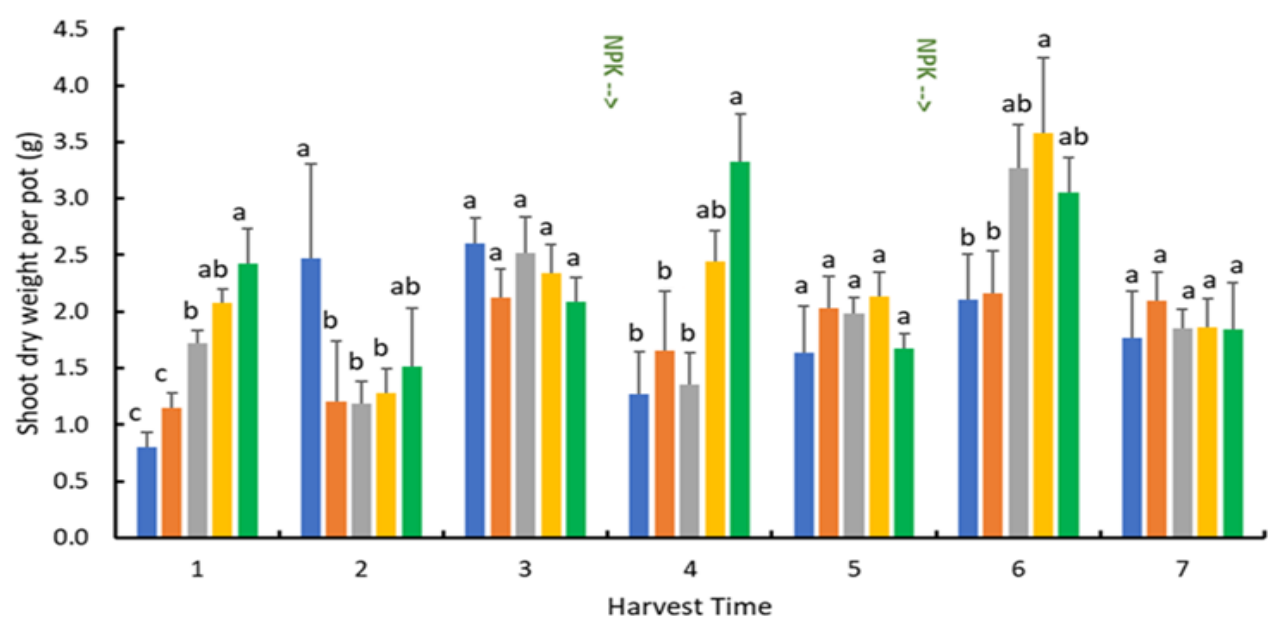

Figure 3. Shoot dry weights on seven consecutive harvests in Ipomoea reptans plant grown at different population densities. Blue, brown, grey, yellow, and green represent $1,2,3,4$, and 5 plants per pot, respectively
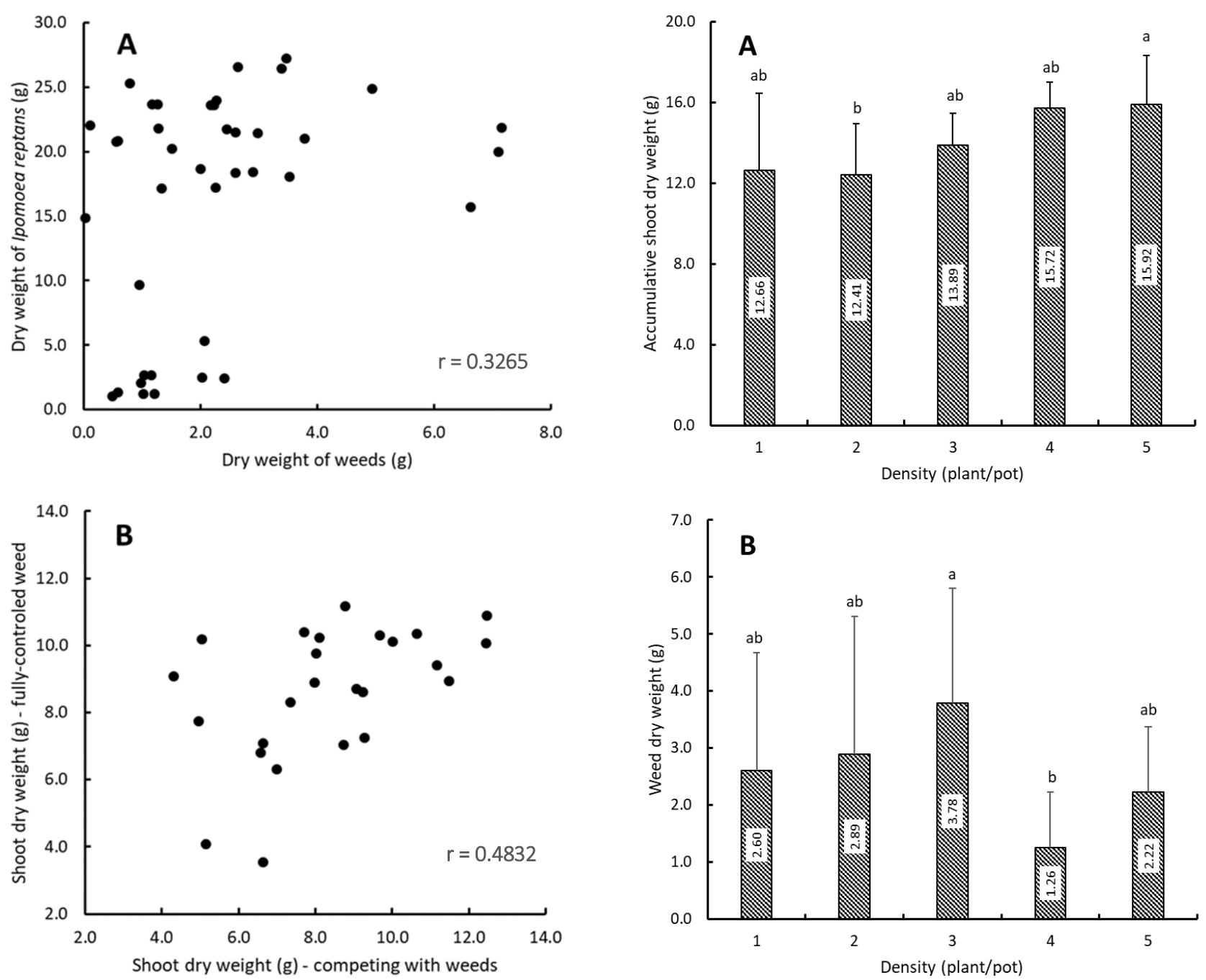

Figure 4. No correlation between dry weight of Ipomoea reptans and weed shoots within each pot (A) and between shoot dry weight of cultivated with or without weed competition (B)

Figure 5. Population density of Ipomoea reptans significantly increased cumulative yield (A) and significantly decreased weed dry weight (B) 

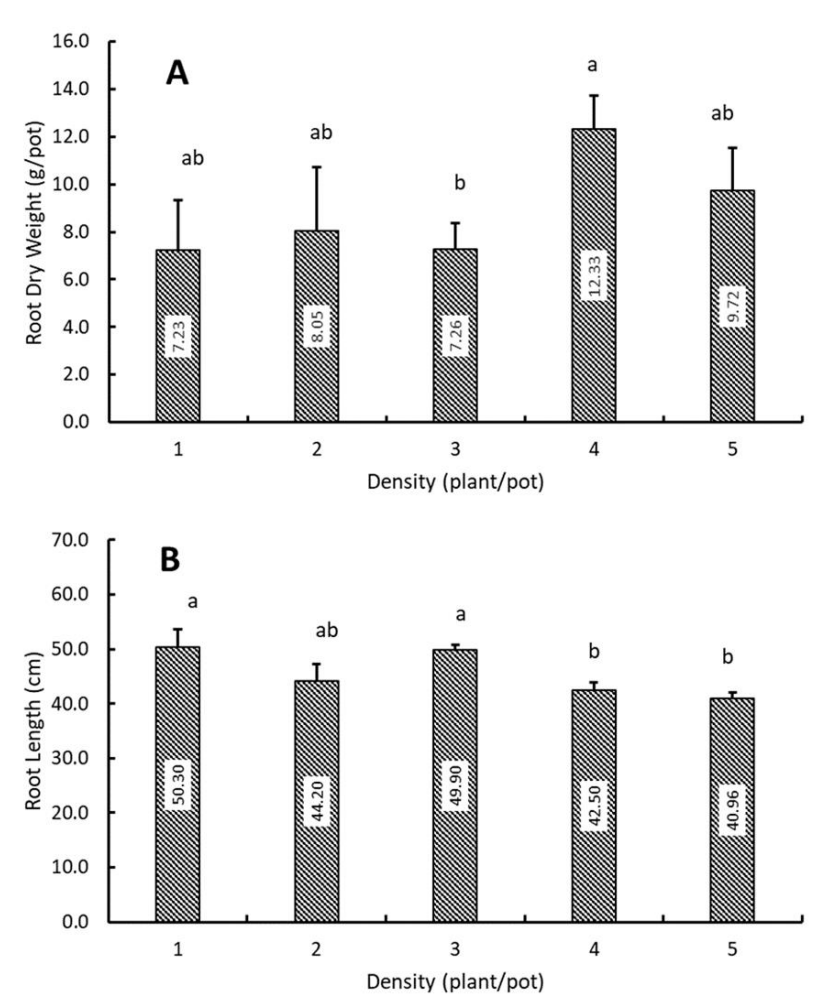

Figure 6. Higher population density increased total root dry weight per pot but the longest root was exhibited by lower population density in Ipomoea reptans

In bottom-wet culture, water was never been a constraint since rhizosphere was continuously wetted by upward water movement from 5-cm saturated layer at base of the perforated pot. However, fertilizers should be regularly applied if multiple harvest system was implemented. Application of NPK fertilizer at rate of $5 \mathrm{~g}$ per pot per week was appropriate for water spinach. Luyen \& Preston (2004) recommended $30 \mathrm{~kg} \mathrm{~N} \mathrm{ha}^{-1}$, equivalent to $65 \mathrm{~kg}$ Urea ha $\mathrm{ha}^{-1}$ for maximum yield in water spinach. Furthermore, Xiang et al. (2020) recommended to use $\mathrm{NH}_{4}$ (ammonium), instead of $\mathrm{NO}_{3}$ (nitrate) in order to minimize nitrogen loss due to leaching.

This research was conducted during dry season. Therefore, depletion of nutrients was mostly due to absorption by plants since water movement within the potted soil was mostly upward due to capillarity force rather than downward due to gravitation. The plants in bottom-wet culture were never been watered from upper surface of the substrate. However, during rainy season, nutrient losses due to leaching could be a problem if the plants were grown in the field or outdoor condition. Zhao et al. (2019) suggested to add biochar in growing substrate for limiting nutrient loss due to leaching.

Overall, across population density treatments, SPAD values were increased at the fourth harvest. The increase was due to reapplication of NPK fertilizer a week earlier. Leaf SPAD value was directly related to nitrogen content (Li et al. 2019; Yue et al. 2020). This is a clear indication of immediate nutrient uptake (within less than a week), especially $\mathrm{N}$, by water spinach in response to the nutrient application. SPAD values were measured base on intensity of green color. Intensity of green color in leafy vegetables is generally preferred by consumers; therefore, high SPAD value can be used as indicator for quality of green leafy vegetables, such as water spinach.

Weed density at rate up to $8 \mathrm{~g}$ dry weight per pot, equivalent to $114 \mathrm{~g} \mathrm{~m}^{-2}$, did not affect yield of water spinach (Figure 4). Water spinach grew vigorously in tropical riparian wetlands and well-adapted to wetland conditions. It grows well on moist soil, shallow soil water table condition, fully water-saturated soil pores, temporary flooding, or completely floating on body of water. In many countries, water spinach is considered as a weed in rice cultivation (Caton et al. 2010), potential feed for fish (Adedokun et al. 2019; Ali \& Kaviraj 2018), but it also has been long known as vegetable in many Asian countries (Yousif et al. 2010). Recently, more studies have been conducted on uses of water spinach as functional food and pharmacological purposes (Chen et al. 2018; Dhanasekaran et al. 2015) and for remediation of chemically polluted lands, especially heavy metals (Tang et al. 2017; Wang et al. 2013).

Water spinach successfully suppressed weed growth as indicated by lower total dry weight of the weeds at higher density of water spinach (Figure 5). Seeds of water spinach germinated earlier and grew faster at earlier vegetative stage. It reached $30 \mathrm{~cm}$ height in about 4 weeks; therefore, late-emerging weeds grew under or between canopies of water spinach. Weeds were unable to compete for light, water, and nutrients under these circumstances. No significant difference in dry weight amongst water spinach grown under controlled and uncontrolled weeds. This finding justified that water spinach was not affected by presence of weeds (Figure 4).

In conclusion, accumulative yields from multiple harvests of 5 consecutive weeks were relatively similar in water spinach cultivated at population densities varied from 1 to 5 plants per pot or equivalent to 14 to 71 plants per $\mathrm{m}^{2}$. Yet, quality of the yield was better in lower population density ( 1 plant per pot or 14 plants per $\mathrm{m}^{2}$ ) as indicated by SPAD value and number of marketable size stems. Weekly NPK fertilizer application at rate of $5 \mathrm{~g}$ per pot was effective in increasing yield as the yield immediately increased after 1 week of application; yet, the beneficial effect was lasting only about a week. First harvest of water spinach can be executed at 4 weeks after seed sowing. This fast-growing water spinach also exhibited ability to compete with native weeds commonly found at tropical wetlands, as its yield was not significantly affected by weed density up to $11.3 \mathrm{mg} \mathrm{cm}^{-2}$.

\section{ACKNOWLEDGEMENTS}

We would like to express our gratitude to all anonymous reviewers for their comments and suggestions. This research was funded by Applied Research Program, Ministry of Research and Technology, Republic of Indonesia, Grant No. 170/SP2H/LT/DRPM/2020, and 
Program Penelitian Unggulan Profesi Universitas Sriwijaya, Grant No. 0687/UN9/SK.BUK.KP/2020.

\section{REFERENCES}

Adedokun MA, Ogundiran MA, Alatise SP. 2019. Preliminary assessment of water spinach (Ipomoea aquatica) and morning glory (Ipomoea asarifolia) leaves meals as non-conventional fish feedstuffs. Int J Fish Aquat Stud 7 (5): 446-450.

Ahmed MN, Mondol MA, Hossain MI, Akter A, Wadud MA. 2013. Performance of kangkong under two years old akashmoni tree. J Agrofor Environ 7: 89-92.

Ali S, Kaviraj A. 2018. Aquatic weed Ipomoea aquatica as feed ingredient for rearing Rohu, Labeo rohita (Hamilton). Egypt J Aquat Res 44 (4): 321-325.

Caton BP, Mortimer M, Hill JE, Johnson DE. 2010. A practical field guide to weeds of Rice in Asia, International Rice Research Institute, Los Banos, Philippines.

Chanu LB, Gupta A. 2016. Phytoremediation of lead using Ipomoea aquatica Forsk. in hydroponic solution. Chemosphere 156: 407-411.

Chen GT, Lu Y, Yang M, Li JL, Fan BY. 2018. Medicinal uses, pharmacology, and phytochemistry of Convolvulaceae plants with central nervous system efficacies: A systematic review. Phytotherapy Res 32 (5): 823-864.

Dhanasekaran S, Perumal P, Palayan M. 2015. In-vitro Screening for acetylcholinesterase enzyme inhibition potential and antioxidant activity of extracts of Ipomoea aquatica Forsk: therapeutic lead for Alzheimer's disease. J Appl Pharm Sci 5 (2): 012-016.

Enriquez VA. 2017. Growth performance of water spinach, Ipomoea aquatica on seaweed, Eucheuma cottonii compost treated soil and other commercial growing media. Int J Agric Econ Dev 5 (1): 29-37.

Easlon HM, Bloom AJ. 2014. Easy Leaf Area: Automated digital image analysis for rapid and accurate measurement of leaf area. Appl Plant Sci 2 (7): 1400033. DOI: 10.3732/apps.1400033.

Guo Z, Wang B, Yin Q, Zhou Y, Xiao J, Jun-Neng L, Huan Z, Luo Y. 2019. Purification effects of floating bed cultivation of water spinach on tilapia aquaculture pond water quality. J Southern Agric 50 (6): 1378-1384.

Hefny-Gad M, Tuenter E, El-Sawi N, Younes S, El-Ghadban EM, Demeyer K, Pieters L, Heyden YV, Mangelings D. 2018. Identification of some bioactive metabolites in a fractionated methanol extract from Ipomoea aquatica (Aerial Parts) through TLC, HPLC, UPLC-ESI-QTOF-MS and LC-SPE-NMR fingerprints analyses. Phytochem Anal 29 (1): 5-15.

Hong J, Gruda NS. 2020. The potential of introduction of Asian vegetables in Europe. Horticulturae 6 (3): 38.

Kaur J, Rawat A, Renu SK, Narain S. 2016. Taxonomy, phytochemistry, traditional uses and cultivation of Ipomoea aquatica Forsk. Imper J Interdisc Res 2 (10): 408-412.

Kriswantoro H, Lakitan B, Lesbani A, Wijaya A. 2020a. Foliar application of 5-aminolevulinic acid for offsetting unfavorable effects of shallow water table on growth and yield in snap bean. Bulg J Agric Sci 26 (3): 638-645.

Kriswantoro H, Lakitan B, Lesbani A, Wijaya A. 2020b. 5-aminolevulinic acid lessened growth suppression in snap bean (Phaseolus vulgaris L.) exposed to shallow water table. Agrivita J Agric Sci 42 (2): 306 319.

Lakitan B, Kadir S, Wijaya A. 2018. Tolerance of common bean (Phaseolus vulgaris L.) to different durations of simulated shallow water table condition. Aust J Crop Sci 12 (4): 661-668.

Lawal U, Leong SW, Shaari K, Ismail IS, Khatib A, Abas F. 2017. $\alpha$-glucosidase inhibitory and antioxidant activities of Different Ipomoea aquatica cultivars and LC-MS/MS profiling of the active cultivar. J Food Biochem 41 (2): e12303. DOI: 10.1111/jfbc.12303.
Li R, Chen J, Qin Y, Fan M. 2019. Possibility of using a SPAD chlorophyll meter to establish a normalized threshold index of nitrogen status in different potato cultivars. J Plant Nutr 42 (8): 834841.

Luyen LT, Preston TR. 2004. Effect of level of urea fertilizer on biomass production of water spinach (Ipomoea aquatica) grown in soil and in water. Livest Res Rural Dev 16 (10): 67-73.

Marin FR, Edreira JIR, Andrade J, Grassini P. 2019. On-farm sugarcane yield and yield components as influenced by number of harvests. Field Crop Res 240: 134-142.

Meihana M, Lakitan B, Susilawati S, Harun MU, Widuri LI, Kartika K, Siaga E, Kriswantoro H. 2017. Steady shallow water table did not decrease leaf expansion rate, specific leaf weight, and specific leaf water content in tomato plants. Aust J Crop Sci 11 (12): 1635-1641.

Rolz C, de León R, de Montenegro ALM, Porras V, Cifuentes R. 2017. A multiple harvest cultivation strategy for ethanol production from sweet sorghum throughout the year in tropical ecosystems. Renew Energy 106: 103-110.

Rondanini DP, Menendez YC, Gomez NV, Miralles DJ, Botto JF. 2017. Vegetative plasticity and floral branching compensate low plant density in modern spring rapeseed. Field Crop Res 210: 104-113.

Saaid MF, Fadhil NSM, Ali MM, Noor MZH. 2013. Automated indoor aquaponic cultivation technique. IEEE 3rd International Conference on System Engineering and Technology, August 2013. DOI: 10.1109/ICSEngT.2013.6650186.

Sarkar RK, Jana JC, Datta S. 2014. Effect of different sowing times and spacings on growth, yield and quality of water spinach (Ipomoea reptans Poir.) under Terai region of West Bengal. J Appl Nat Sci 6 (2): 489-494.

Selamat A, Atiman SA, Puteh A, Abdullah NAP, Mohamed MTM, Zulkeefli AA, Othman S. 2012. Allometry deterministic approaches in cell size, cell number and crude fiber content related to the physical quality of kangkong (Ipomoea reptans) grown under different plant density pressures. Int J Modern Physic 9: 30-43.

Susilawati S, Lakitan B. 2019. Cultivation of common bean (Phaseolus vulgaris L.) subjected to shallow water table at riparian wetland in South Sumatra, Indonesia. Aust J Crop Sci 13 (1): 98-104.

Tang L, Luo W, Chen W, He Z, Gurajala HK, Hamid Y, Deng M, Yang X. 2017. Field crops (Ipomoea aquatica Forsk. and Brassica chinensis L.) for phytoremediation of cadmium and nitrate cocontaminated soils via rotation with Sedum alfredii Hance. Environ Sci Pollut Res 24 (23): 19293-19305.

Wang S, Zhou X, Chu J, Shi Y. 2013. Pollutant removal efficiency by using compound systems of Ipomoea aquatica ecological floating bed and bionic macrophytes. Environ Sci Tech 36 (3): 78-82.

Wang W, He A, Jiang G, Sun H, Jiang M, Man J, Ling X, Cui K, Huang J, Peng S, Nie L. 2020. Ratoon rice technology: A green and resourceefficient way for rice production. Adv Agron 159: 135-167.

Xiang S, Wu S, Zhang Q, Liu Y, Ruan R. 2020. A nitrogen dynamic hydroponic culture on performance and quality of water spinach (Ipomoea aquatica). J Plant Nutr 43 (6): 773-783.

Yousif BS, Nguyen NT, Fukuda Y, Hakata H, Okamoto Y, Masaoka Y, Saneoka H. 2010. Effect of salinity on growth, mineral composition, photosynthesis and water relations of two vegetable crops; New Zealand spinach (Tetragonia tetragonioides) and water spinach (Ipomoea aquatica). Int J Agric Biol 12 (2): 211-216.

Yue X, Hu Y, Zhang H, Schmidhalter U. 2020. Evaluation of both SPAD reading and SPAD index on estimating the plant nitrogen status of winter wheat. Int J Plant Prod 14: 67-75.

Zhang Q, Achal V, Xu Y, Xiang WN. 2014. Aquaculture wastewater quality improvement by water spinach (Ipomoea aquatica Forsskal) floating bed and ecological benefit assessment in ecological agriculture district. Aquacult Eng 60: 48-55.

Zhao F, Zou G, Shan Y, Ding Z, Dai M, He Z. 2019. Coconut shell derived biochar to enhance water spinach (Ipomoea aquatica Forsk) growth and decrease nitrogen loss under tropical conditions. Sci Rep 9 (1): 20291 DOI: 10.1038/s41598-019-56663-w. 